\title{
Large-scale thermal convection in a horizontal porous layer
}

\author{
Denis S. Goldobin ${ }^{1,2}$ and Elizaveta V. Shklyaeva ${ }^{1}$ \\ ${ }^{1}$ Department of Theoretical Physics, Perm State University, 15 Bukireva str., 614990, Perm, Russia \\ ${ }^{2}$ Department of Physics and Astronomy, University of Potsdam, Postfach 601553, D-14415 Potsdam, Germany
}

\begin{abstract}
In a range of physical systems the first instability in Rayleigh-Bérnard convection between nearly thermally insulating horizontal plates is large-scale. This holds for thermal convection of fluids saturating porous media. Large-scale thermal convection in a horizontal layer is governed by remarkably similar equations both in the presence of a porous matrix and without it, with only one additional term for the latter case, which, however, vanishes under certain conditions (e.g., two-dimensional flows or infinite Prandtl number). We provide a rigorous derivation of long-wavelength equations for a porous layer with inhomogeneous heating and possible pumping.
\end{abstract}

PACS numbers: $44.25 .+\mathrm{f}, 44.30 .+\mathrm{v}, 47.54 .-\mathrm{r}$

Rayleigh-Bérnard convection, i.e., a thermal buoyancy convection in a layer confined between two rigid horizontal plates and uniformly heated from below, is one of the classical problems of fluid dynamics. When the bounding plates are nearly thermally insulating, the first instability of the heat conductive state (quiescent state) of the layer is long-wavelength (large-scale) [1, 2, 3, 4] , i.e., horizontal gradients of the fluid velocity field are small compared to vertical ones and, thus, the horizontal scale of the flow is large compared to the layer height. For large-scale patterns, temperature perturbations $\theta$ are nearly uniform along the vertical coordinate $z$ and in terms of $\theta(x, y)$ the problem is two-dimensional (2D). Making allowance for the heating inhomogeneity, one can find

$$
\begin{gathered}
\partial_{t} \theta+\Delta^{2} \theta-\nabla \cdot(\theta \vec{s})+\nabla \cdot\left[q(x, y) \nabla \theta-\nabla \theta|\nabla \theta|^{2}\right]=0 \\
\Delta \vec{s}=0, \quad[\nabla \times \vec{s}]_{z} \equiv B=\frac{3}{2 P r}[\nabla \times(\nabla \theta \Delta \theta)]_{z}
\end{gathered}
$$

Here $\partial_{t}$ denotes the partial time derivative, $q(x, y)$ is the relative departure of the heat flux (imposed by the heating) through the boundaries from the critical value for a homogeneous heating, Prandtl number $\operatorname{Pr}=\nu / \chi$ is the ratio of the kinematic viscosity and the heat diffusivity, $\vec{s}$ is responsible for advectional heat transfer. Knobloch [5] has discussed pattern selection for Eq. (1) with uniform $q$ and $\vec{s}=0$, which is relevant, e.g., to $\operatorname{Pr} \rightarrow \infty$ or $2 \mathrm{D}$ setups where flows are homogeneous in the $y$-direction. Later on, the term missing in [5] was accounted for in [6]. Remarkably, in 7], equations similar to (11) have been obtained for large-scale turbulence in Rayleigh-Bérnard convection.

Various problems related to large-scale thermal convection in porous media have been repeatedly addressed in the literature (e.g., 3, 4]). However, researches typically either deal with the case of a rectangular cavity with a large aspect ratio ([3], where no equations similar to (12) have been derived) or discuss a finite departure from the stability threshold ([4]; such a departure leads to discontinuities of the velocity field, which cannot be treated within the framework of the long-wavelength approximation). Pattern formation under heating inhomogeneity has been rather extensively studied for thin films (e.g., 8] and Refs. therein) owing to important technical applications (rupture of lubricating films 9], etc.). To the authors' knowledge, for porous media the equation system similar to (12) has not been presented in the literature.

In this Brief Report, we introduce the specific physical system we deal with. Then we derive the equation of large-scale convection for this system and find that it is similar to Eqs. (12) with $B=0$, though the relationships between the flow and the temperature perturbation are different for different fluid dynamical systems [4, 6, 7].

a. Thermal convection in a horizontal porous layer. Let us consider convection of a fluid saturating a horizontal porous layer heated from below. Boundaries are impermeable, the heat flux across the layer is fixed (implying the heat diffusivity of the boundaries is small compared to the one of the porous matrix saturated with the fluid), but inhomogeneous along the layer. Relaxation of the local temperature difference between the porous matrix and the fluid is assumed to be fast, and we do not introduce several temperatures for them. For small perturbations of the temperature about $T_{0}$, one may guess a linear dependence of the fluid density on the temperature; $\rho(T)=\rho_{0}\left(1-\beta\left(T-T_{0}\right)\right)$, where $\rho_{0}=\rho\left(T_{0}\right)$, $\beta=(\partial \rho / \partial T)_{p}$. The reference frame is such that the $(x, y)$-plane is horizontal, $z=0$ and $z=h$ are the lower and upper layer boundaries, respectively. We adopt the conventional Darcy-Boussinesq approximation [2],

$$
\begin{gathered}
0=-\rho_{0}^{-1} \nabla p-\nu K^{-1} \vec{v}+g \beta T \vec{e}_{z} \\
\partial_{t} T+b^{-1} \nabla \cdot(\vec{v} T)=\chi \Delta T \\
\nabla \cdot \vec{v}=0 \\
z=0, h: \quad v_{z}=0, \quad \partial_{z} T=-A(1+q(x, y)),
\end{gathered}
$$

where $\vec{v}$ : the macroscopic filtration velocity, $K$ : the permeability, $\vec{g}=-g \vec{e}_{z}$ : the gravity, $b$ : the specific heat capacity of the saturated porous medium divided by the one of the fluid, $\chi$ : the heat diffusivity of the saturated porous medium, $\chi C_{p} A(1+q(x, y))$ : the imposed heat flux ( $C_{p}$ is the specific heat capacity).

It is convenient to measure the length by layer height $h$, time by $h^{2} / \chi$, the velocity by $b \chi / h$, the temperature by $A h$, and the pressure by $b \rho_{0} \nu \chi / K$. The dimensionless 
parameter governing the behavior of the system is the Rayleigh-Darcy number $R=\beta A h^{2} g K / b \nu \chi$.

The dimensionless form of system (36) reads

$$
\begin{gathered}
-\nabla p-\vec{v}+R T \vec{e}_{z}=0, \\
\partial_{t} T+\nabla \cdot(\vec{v} T)=\Delta T, \\
\nabla \cdot \vec{v}=0, \\
z=0,1: \quad v_{z}=0, \quad \partial_{z} T=-1-q(x, y) .
\end{gathered}
$$

b. Long-wavelength approximation. For a uniform fixed heat flux the first instability is known to be longwavelength [2]. This holds for $q(x, y)$ slowly varying in the boundary plane: $|\nabla q| /|q| \sim \varepsilon \ll 1$. In order to avoid large temperature gradients, which correspond to jumps of derivatives of fields in the long-wavelength limit [4], we restrict ourselves to a small supercriticality. Below we will find the critical value $R_{\mathrm{C}}$ for a homogeneous heating and set $R=R_{\mathrm{C}}$; hence, nearcritical regimes occur for small $q(x, y)$ which may be thought to measure the relative departure of the Rayleigh-Darcy number from the critical value.

Owing to Eq. (9) the horizontal component of velocity $\vec{v}$ is large compared to the vertical one because variations of velocity $\vec{v}$ by shifts along the horizontal directions are small compared to ones by shifts transversal to the layer. Let us explicitly account for this fact, $\vec{v}=w \vec{e}_{z}+\varepsilon^{-1} \vec{u}$, where $\vec{u}$ is the horizontal component of the filtration velocity field, $w$ is the vertical one. Rescaling horizontal coordinates, $(x, y) \rightarrow\left(\varepsilon^{-1} x, \varepsilon^{-1} y\right)$, explicitly writing $q(x, y)=\varepsilon^{2} q_{2}(x, y)$, and projecting momentum conservation law (7) onto the vertical and horizontal directions, one may rewrite equation system (7) 10) in a form convenient for the further treatment;

$$
\begin{gathered}
-\partial_{z} p-w+R T=0 \\
\vec{u}=-\varepsilon^{2} \nabla_{2} p \\
\partial_{t} T+\partial_{z}(w T)+\nabla_{2} \cdot(\vec{u} T)=\partial_{z}^{2} T+\varepsilon^{2} \Delta_{2} T \\
\partial_{z} w+\nabla_{2} \cdot \vec{u}=0 \\
z=0,1: \quad w=0, \quad \partial_{z} T=-1-\varepsilon^{2} q_{2}(x, y) .
\end{gathered}
$$

Here the subscript "2" for spatial derivatives means the differentiation with respect to two horizontal coordinates.

Since $\varepsilon$ appears squared in (11) 15), only even powers of $\varepsilon$ are present in the expansion: $w=w_{0}+\varepsilon^{2} w_{2}+\varepsilon^{4} w_{4}+$ $\ldots, T=T_{0}+\varepsilon^{2} T_{2}+\varepsilon^{4} T_{4}+\ldots$, etc. The long-wavelength approximation assumes a weak spatial inhomogeneity of temperature perturbations which results in a slow temporal evolution. With only even powers of $\varepsilon$ in the expansion, one expects characteristic times of the evolution of large-scale patterns to be not lesser than $\propto \varepsilon^{-2}$; therefore, in terms of "slow" times, $\partial_{t}=\varepsilon^{2} \partial_{t_{2}}+\varepsilon^{4} \partial_{t_{4}}+\ldots$ $\underline{\varepsilon^{0}}$ : In the leading order, problem (11) 15) yields

$$
\begin{gathered}
-\partial_{z} p_{0}-w_{0}+R T_{0}=0, \\
\vec{u}_{0}=0, \\
\partial_{z}\left(w_{0} T_{0}\right)+\nabla_{2} \cdot\left(\vec{u}_{0} T_{0}\right)=\partial_{z}^{2} T_{0}, \\
\partial_{z} w_{0}+\nabla_{2} \cdot \vec{u}_{0}=0, \\
z=0,1: \quad w_{0}=0, \quad \partial_{z} T_{0}=-1 .
\end{gathered}
$$

From Eqs. (1917), $\partial_{z} w_{0}=0, w_{0}=C_{1}(x, y)=0$ $\left[C_{1}=0\right.$ due to the boundary conditions (BCs)]. From Eq. (18), $\partial_{z}^{2} T_{0}=0$, i.e., $T_{0}=C_{2}(x, y) z+\theta(x, y)$; accounting for BCs (20), one obtains

$$
T_{0}=-z+\theta(x, y),
$$

where $\theta(x, y)$ should be determined from higher orders of the expansion while here it appears as an unknown function of the horizontal coordinates.

From Eq. (16), $\partial_{z} p_{0}=R T_{0}=-R z+R \theta(x, y)$,

$$
p_{0}=-\frac{1}{2} R z^{2}+R \theta(x, y) z+\Pi_{0}(x, y),
$$

where $\Pi_{0}(x, y)$ is unknown in this order of the expansion.

$$
\begin{gathered}
-\partial_{z} p_{2}-w_{2}+R T_{2}=0 \\
\vec{u}_{2}=-\nabla_{2} p_{0}, \\
\partial_{t_{2}} T_{0}+\partial_{z}\left(w_{2} T_{0}\right)+\nabla_{2} \cdot\left(\vec{u}_{2} T_{0}\right)=\partial_{z}^{2} T_{2}+\Delta_{2} T_{0}, \\
\partial_{z} w_{2}+\nabla_{2} \cdot \vec{u}_{2}=0 \\
z=0,1: \quad w_{2}=0, \quad \partial_{z} T_{2}=-q_{2}(x, y) .
\end{gathered}
$$

From Eq. (24),

$$
\vec{u}_{2}=-\nabla_{2} p_{0}=-R z \nabla_{2} \theta(x, y)-\nabla_{2} \Pi_{0}(x, y) .
$$

From Eq. (26),

$$
\begin{aligned}
& \partial_{z} w_{2}=-\nabla_{2} \cdot \vec{u}_{2}=R z \Delta_{2} \theta(x, y)+\Delta_{2} \Pi_{0}(x, y), \\
& w_{2}=\frac{1}{2} R z^{2} \Delta_{2} \theta(x, y)+\Delta_{2} \Pi_{0}(x, y) z+C_{3}(x, y) .
\end{aligned}
$$

BCs (27) for the velocity field yield

$$
\begin{aligned}
& z=0: C_{3}=0 \\
& z=1: \Delta_{2} \Pi_{0}(x, y)=-\frac{1}{2} R \Delta_{2} \theta(x, y) .
\end{aligned}
$$

From the latter BC,

$$
\Pi_{0}(x, y)=-\frac{1}{2} R \theta(x, y)+\pi_{0}(x, y), \quad \Delta_{2} \pi_{0}(x, y)=0 .
$$

Note, $\left\langle\vec{u}_{2}\right\rangle=-\nabla_{2} \pi_{0}$ (henceforth, $\langle f\rangle \equiv \int_{0}^{1} f \mathrm{~d} z$ ). Let us consider a layer domain limited in the horizontal directions by boundary $\Gamma$ (to keep it simple, we assume $\Gamma$ to be vertical), where a fixed fluid gross flux (or its absence; "gross" means averaged over $z$, and, in particular, the absence of the gross flux does not necessarily claim the absence of the flux) is imposed. Mathematically, this means that the orthogonal to $\Gamma$ component of $\left\langle\vec{u}_{2}\right\rangle$ is fixed: $\left\langle\vec{u}_{2}\right\rangle_{n}=Q(Q$ is not uniform along $\Gamma$; owing to mass conservation, $\left.\int_{\Gamma} Q \mathrm{~d} \Gamma=0\right)$. Hence, one obtains the boundary problem for $\pi_{0}(x, y)$,

$$
\Delta_{2} \pi_{0}=0,\left.\quad \partial_{n} \pi_{0}\right|_{\Gamma}=-Q \quad\left(\int_{\Gamma} Q \mathrm{~d} \Gamma=0\right),
$$

where $\partial_{n}$ is the orthogonal to $\Gamma$ component of the gradient. This problem has a unique solution (up to an insignificant constant) unambiguously defined by $Q$. Thus, $\pi_{0}(x, y)$ describes an imposed advection (pumping) in the layer, which is caused and unambiguously controlled by BCs on $\Gamma$ (the pressure or the fixed gross flux). As soon 
as we consider a nearcritical behavior, it makes sense to not allow for an imposed advection in the leading order, otherwise a moderately strong advection would overpress the effect of a weakly inhomogeneous heating. We set

$$
\pi_{0}(x, y)=0
$$

and will take the imposed advection into account in higher orders.

With (29), one can write down

$$
\begin{aligned}
& \vec{u}_{2}=-R\left(z-\frac{1}{2}\right) \nabla_{2} \theta(x, y), \\
& w_{2}=\frac{1}{2} R\left(z^{2}-z\right) \Delta_{2} \theta(x, y) .
\end{aligned}
$$

Let us now integrate (25) over $z \in[0,1]$;

$\partial_{t_{2}}\left\langle T_{0}\right\rangle+\left\langle\partial_{z}\left(w_{2} T_{0}\right)\right\rangle+\nabla_{2} \cdot\left\langle\vec{u}_{2} T_{0}\right\rangle=\left\langle\partial_{z}^{2} T_{2}\right\rangle+\Delta_{2}\left\langle T_{0}\right\rangle$.

Due to BCs (27), $\int_{0}^{1} \partial_{z}\left(w_{2} T_{0}\right) \mathrm{d} z=\left.w_{2} T_{0}\right|_{z=0} ^{1}=0$, $\int_{0}^{1} \partial_{z}^{2} T_{2} \mathrm{~d} z=\left.\partial_{z} T_{2}\right|_{z=0} ^{1}=-\left.q_{2}(x, y)\right|_{z=0} ^{1}=0$. Whereas,

$$
\nabla_{2} \cdot\left\langle\vec{u}_{2} T_{0}\right\rangle=-\nabla_{2} \cdot\left\langle\vec{u}_{2} z\right\rangle+\nabla_{2} \cdot\left(\left\langle\vec{u}_{2}\right\rangle \theta\right) .
$$

Substituting (30) into the first term of the rhs of the last equation and accounting for $\left\langle\vec{u}_{2}\right\rangle=-\nabla_{2} \pi_{0}=0$, one finds

$$
\nabla_{2} \cdot\left\langle\vec{u}_{2} T_{0}\right\rangle=R \Delta_{2} \theta\left\langle z^{2}-z / 2\right\rangle=(R / 12) \Delta_{2} \theta .
$$

For the rest of the terms in Eq. (32), $\partial_{t_{2}} T_{0}=\partial_{t_{2}} \theta$, $\Delta_{2} T_{0}=\Delta_{2} \theta$, and Eq. (32) finally reads

$$
\partial_{t_{2}} \theta=(1-R / 12) \Delta_{2} \theta \text {. }
$$

For $R<12$, Eq. (33) is a conventional diffusion equation, and for trivial BCs (on $\Gamma$ ) or an infinite layer, all inhomogeneities of $\theta$ decay. For $R>12$, it is a diffusion equation with a negative diffusivity, where all the inhomogeneous perturbations grow. Thus, $R=12$ is the linear stability threshold of the system. Note, nonlinearity does not play a role in this order of the expansion. In order to account for nonlinear effects and the dependence of the linear stability on the wavelength (now all the perturbations either grow or decay regardless to their wavelength), we should restrict ourselves to the vicinity of the stability threshold. For this purpose, we set

$$
R=R_{\mathrm{C}}=12
$$

and introduce departure from the threshold via $q$. Since the local Rayleigh-Darcy number ("local" means defined for a small domain of the layer) $R_{\text {local }}=R(1+q)$, positive $q$ corresponds to a supercritical regime, negative $q$ does to a subcritical one. As soon as $R=12, \partial_{t_{2}} \theta=0$; therefore, we should consider a slower evolution, $\partial_{t} \theta=\varepsilon^{4} \partial_{t_{4}} \theta+\ldots$.

Let us now derive $T_{2}$ from Eq. (25).

$$
\begin{aligned}
& \quad \partial_{z}^{2} T_{2}=\partial_{z}\left(w_{2} T_{0}\right)+\nabla_{2} \cdot\left(\vec{u}_{2} T_{0}\right)-\Delta_{2} T_{0} \\
& T_{2}=-\left(\frac{3}{2} z^{4}-2 z^{3}\right) \Delta_{2} \theta+\left(2 z^{3}-3 z^{2}\right) \theta \Delta_{2} \theta \\
& \quad+\left(z^{4}-z^{3}\right) \Delta_{2} \theta-\left(2 z^{3}-3 z^{2}\right) \nabla_{2} \cdot\left(\theta \nabla_{2} \theta\right)-\frac{1}{2} z^{2} \Delta_{2} \theta \\
& \quad+C_{4}(x, y) z+\theta_{2}(x, y) .
\end{aligned}
$$

Due to the relation $\nabla_{2} \cdot\left(\theta \nabla_{2} \theta\right)=\theta \Delta_{2} \theta+\left(\nabla_{2} \theta\right)^{2}$, one can eliminate the term $\theta \Delta_{2} \theta$ from the expression for $T_{2}$;

$$
\begin{aligned}
T_{2} & =\left(-\frac{1}{2} z^{4}+z^{3}-\frac{1}{2} z^{2}\right) \Delta_{2} \theta \\
& +\left(-2 z^{3}+3 z^{2}\right)\left(\nabla_{2} \theta\right)^{2}+C_{4}(x, y) z+\theta_{2}(x, y)
\end{aligned}
$$

Owing to BCs (27), $C_{4}(x, y)=-q_{2}(x, y) ; \theta_{2}(x, y)$ is still undefined.

Remarkably, $\theta$ and $\theta_{2}$ depend on $z$ in one and the same fashion (i.e., are uniform along $z$ ) and are for the moment undetermined functions of $x$ and $y$. Hence, $\theta_{2}$ may be chosen as one needs, and this will be automatically balanced by $\theta$. Let us use this fact. Notice,

$$
\langle T\rangle=-\frac{1}{2}+\theta(x, y)+\varepsilon^{2}\left\langle T_{2}\right\rangle+O\left(\varepsilon^{4}\right) ;
$$

therefore, if one defines $\theta_{2}$ in such a way as to make $\left\langle T_{2}\right\rangle=0$, then $\theta$ will be a $z$-mean temperature up to the truncation accuracy of our expansion, i.e., $\varepsilon^{4}$. Thus,

$$
\begin{gathered}
\left\langle T_{2}\right\rangle=-\frac{1}{60} \Delta_{2} \theta+\frac{1}{2}\left(\nabla_{2} \theta\right)^{2}-\frac{1}{2} q_{2}(x, y)+\theta_{2}(x, y)=0 \\
\theta_{2}=\frac{1}{60} \Delta_{2} \theta-\frac{1}{2}\left(\nabla_{2} \theta\right)^{2}+\frac{1}{2} q_{2} .
\end{gathered}
$$

Finally,

$$
\begin{aligned}
T_{2}= & \left(-\frac{1}{2} z^{4}+z^{3}-\frac{1}{2} z^{2}+\frac{1}{60}\right) \Delta_{2} \theta \\
& +\left(-2 z^{3}+3 z^{2}-\frac{1}{2}\right)\left(\nabla_{2} \theta\right)^{2}+\left(-z+\frac{1}{2}\right) q_{2} .
\end{aligned}
$$

From Eq. (23) follows $\partial_{z} p_{2}=-w_{2}+R T_{2}$, and, integrating it with respect to $z$, one finds the pressure

$$
\begin{aligned}
p_{2}= & \left(-\frac{6}{5} z^{5}+3 z^{4}-4 z^{3}+3 z^{2}+\frac{1}{5} z\right) \Delta_{2} \theta \\
& +\left(-6 z^{4}+12 z^{3}-6 z\right)\left(\nabla_{2} \theta\right)^{2} \\
& +\left(-6 z^{2}+6 z\right) q_{2}+\Pi_{2}(x, y) .
\end{aligned}
$$

$\varepsilon^{4}$ : As we will not construct the expansion beyond this order, we do not have to consider all the equations. The following is sufficient;

$$
\begin{gathered}
\vec{u}_{4}=-\nabla_{2} p_{2} \\
\partial_{t_{4}} T_{0}+\partial_{z}\left(w_{4} T_{0}\right)+\nabla_{2} \cdot\left(\vec{u}_{4} T_{0}\right) \\
+\partial_{z}\left(w_{2} T_{2}\right)+\nabla_{2} \cdot\left(\vec{u}_{2} T_{2}\right)=\partial_{z}^{2} T_{4}+\Delta_{2} T_{2} \\
\partial_{z} w_{4}+\nabla_{2} \cdot \vec{u}_{4}=0 \\
z=0,1: \quad w_{4}=0, \quad \partial_{z} T_{4}=0
\end{gathered}
$$

Without calculating $\vec{u}_{4}$, we may substitute (38) directly into (40); $\partial_{z} w_{4}=\Delta_{2} p_{2}$. Then

$$
\begin{aligned}
w_{4} & =\left(-\frac{1}{5} z^{6}+\frac{3}{5} z^{5}-z^{4}+z^{3}+\frac{1}{10} z^{2}\right) \Delta_{2}^{2} \theta \\
& +\left(-\frac{6}{5} z^{5}+3 z^{4}-3 z^{2}\right) \Delta_{2}\left(\nabla_{2} \theta\right)^{2} \\
& +\left(-2 z^{3}+3 z^{2}\right) \Delta_{2} q_{2}+z \Delta_{2} \Pi_{2} .
\end{aligned}
$$

From BCs (41),

$$
\left.w_{4}\right|_{z=1}=0=\frac{1}{2} \Delta_{2}^{2} \theta-\frac{6}{5} \Delta_{2}\left(\nabla_{2} \theta\right)^{2}+\Delta_{2} q_{2}+\Delta_{2} \Pi_{2} ;
$$

therefore,

$$
\Pi_{2}=-\frac{1}{2} \Delta_{2} \theta+\frac{6}{5}\left(\nabla_{2} \theta\right)^{2}-q_{2}+\pi_{2},
$$


where $\Delta_{2} \pi_{2}=0$.

Notice, making use of Eq. (37), one can calculate

$$
\left\langle\vec{u}_{4}\right\rangle=-\nabla_{2}\left\langle p_{2}\right\rangle=-\nabla_{2} \pi_{2}(x, y) .
$$

We have already established the relationship between $\left\langle\vec{u}_{2}\right\rangle$ and $\pi_{0}$ and found $\pi_{0}$ to describe an imposed advection. We have set $\pi_{0}=0$, but now it makes sense to keep $\pi_{2}$, since it describes an advection imposed by lateral boundary conditions.

Substituting $\Pi_{2}$ into Eq. (42), one obtains the final expression

$$
\begin{gathered}
w_{4}=\left(-\frac{1}{5} z^{6}+\frac{3}{5} z^{5}-z^{4}+z^{3}+\frac{1}{10} z^{2}-\frac{1}{2} z\right) \Delta_{2}^{2} \theta \\
+\left(-\frac{6}{5} z^{5}+3 z^{4}-3 z^{2}+\frac{6}{5} z\right) \Delta_{2}\left(\nabla_{2} \theta\right)^{2} \\
+\left(-2 z^{3}+3 z^{2}-z\right) \Delta_{2} q_{2} .
\end{gathered}
$$

Now the integration of Eq. (39) over $z \in[0,1]$ yields the evolution equation for $\theta$

$$
\begin{aligned}
\partial_{t_{4}} \theta & +\left\langle\partial_{z}\left(w_{4} T_{0}\right)\right\rangle+\nabla_{2} \cdot\left\langle\vec{u}_{4} T_{0}\right\rangle \\
& +\left\langle\partial_{z}\left(w_{2} T_{2}\right)\right\rangle+\nabla_{2} \cdot\left\langle\vec{u}_{2} T_{2}\right\rangle=\left\langle\partial_{z}^{2} T_{4}\right\rangle+\Delta_{2}\left\langle T_{2}\right\rangle .
\end{aligned}
$$

The mean values of all $z$-derivatives are zero due to BCs; additionally, $\left\langle T_{2}\right\rangle=0$. The remainder is

$$
\partial_{t_{4}} \theta+\nabla_{2} \cdot\left\langle\vec{u}_{4} T_{0}+\vec{u}_{2} T_{2}\right\rangle=0 .
$$

With $T_{0}, T_{2}, p_{2}$, and $\vec{u}_{2}$ known, one can find

$$
\begin{aligned}
& \nabla_{2} \cdot\left\langle\vec{u}_{4} T_{0}\right\rangle=-\nabla_{2} \cdot\left\langle T_{0} \nabla_{2} p_{2}\right\rangle=\frac{2}{21} \Delta_{2}^{2} \theta-\nabla_{2} \cdot\left(\theta \nabla_{2} \pi_{2}\right) ; \\
& \nabla_{2} \cdot\left\langle\vec{u}_{2} T_{2}\right\rangle=-\nabla_{2} \cdot\left(\frac{6}{5} \nabla_{2} \theta\left(\nabla_{2} \theta\right)^{2}-q_{2} \nabla_{2} \theta\right) .
\end{aligned}
$$

Substituting (45) and (46) into Eq. (44), one obtains the slow evolution equation for $\theta$ in the final form which is similar to Eq. (1);

$$
\partial_{t} \theta+\vec{U} \cdot \nabla \theta+\frac{2}{21} \Delta^{2} \theta-\nabla \cdot\left(\frac{6}{5} \nabla \theta(\nabla \theta)^{2}-q \nabla \theta\right)=0 .
$$

Here the imposed advection $\vec{U} \equiv-\nabla \pi=\langle\vec{u}\rangle, \nabla \cdot \vec{U}=0$, subscripts "2" for differential operators are omitted as all the fields depend on $x$ and $y$ only, indexes indicating the smallness order are also omitted as this equation remains valid in original terms (without formal smallness parameter $\varepsilon$ ). From Eqs. (30|31) the fluid flow up to the leading order of accuracy is

$$
\vec{v}=6(1-2 z) \nabla \theta(x, y)+6\left(z^{2}-z\right) \Delta \theta(x, y) \vec{e}_{z} .
$$

Advection speed $U \propto \varepsilon^{3}$ is small against $u \propto \varepsilon$ when flow is excited, but is important due to its properties: in contrast to $\vec{u}, \vec{U}$ provides a nonzero gross fluid flux through a vertical cross-section of the layer.

Note, the heating inhomogeneity makes the quiescent state impossible, and from Eqs. (36) and (43) it follows that below the convective instability threshold, when $\theta$ decays to zero, the establishing state has nontrivial

$w_{\mathrm{BG}}=\left(-2 z^{3}+3 z^{2}-z\right) \Delta q, \quad T_{\mathrm{BG}}=\left(\frac{1}{2}-z\right) q-z .(48)$ However, these fields are small against the fields excited above the threshold.

Remarkably, Eq. (47) holds for the case of a uniform heating and a weakly inhomogeneous porous matrix. In the most general case, $q(x, y)$ should be replaced with

$$
q_{\mathrm{gen}}(x, y)=q(x, y)+\frac{K(x, y)}{b(x, y) \chi(x, y)} \frac{b_{0} \chi_{0}}{K_{0}}-1
$$

[meanwhile, the background state (48) for this general case cannot be obtained via the plain replacement of $q$ by $\left.q_{\text {gen }}\right]$. This remark is important for experiments as it is more convenient to maintain/control a uniform heating of the layer with a weak inhomogeneity of the structure of the porous matrix, which is inevitable in real systems. Owing to the same reason, the physical system we have discussed is the most relevant one for works [10] addressing phenomena related to spatially localized convective currents excited under parametric disorder [frozen randomly inhomogeneous $q=q(x)$ ].

DG acknowledges the Foundation "Perm Hydrodynamics," CRDF (Grant no. Y5-P-09-01), and MESRF (Grant no. 2.2.2.3.16038) for financial support.
[1] E. M. Sparrow, R. J. Goldstein, and V. K. Jonsson, J. Fluid Mech. 18, 513 (1964).

[2] Transport Phenomena in Porous Media, edited by D. B. Ingham and I. Pop (Pergamon, Oxford, 1998).

[3] K. L. Walker and G. M. Homsy, J. Fluid Mech. 87, 449 (1978); P. G. Daniels, P. G. Simpkins, and P. A. Blythe, Proc. R. Soc. A 426, 107 (1989); P. G. Daniels, J. Fluid Mech. 565, 441 (2006); A. Bahloul, N. Boutana, and P. Vasseur, ibid. 491, 325 (2003).

[4] D. S. Goldobin and D. V. Lyubimov, JETP 104, 830 (2007).

[5] E. Knobloch, Physica D 41, 450 (1990).

[6] L. Shtilman and G. Sivashinsky, Physica D 52, 477 (1991).
[7] S. N. Aristov and P. G. Frick, Fluid Dyn. 24, 960 (1989).

[8] L. Y. Yeo, R. V. Craster, and O. K. Matar, Phys. Rev. E 67, 056315 (2003).

[9] M. R. E. Warner, R. V. Craster, and O. K. Matar, Phys. Fluids 14, 1642 (2002); A. Oron, S. H. Davis, and S. G. Bankoff, Rev. Mod. Phys. 69, 931 (1997).

[10] D. S. Goldobin and E. V. Shklyaeva, Localization and advectional spreading of convective flows under parametric disorder, Phys. Rev. E (submitted, 2008) arXiv:0804.3741; Diffusion of a passive scalar by convective flows under parametric disorder, J. Stat. Mech. (preprint, 2008) arXiv:0805.1518. 